The Amygdaloids performed tunes from their new album, Brainstorm, some of which explore how love can disrupt normal thought processing. In Crime of Passion, a jealous lover's rage momentarily overwhelms his rational thinking: "Sentenced to death for a crime I did commit/I couldn't stop/I did it in a fit/of anger and pain."

Cognitive psychologist, vocalist and guitarist Daniel Levitin of McGill University in Montreal, Canada, sang a scientific homage to the 1989 hit Wicked Game in which Chris Isaak croons "No, I don't want to fall in love." Levitin's version,
I Don't Want My Brain Cut In Two, refers to a drastic procedure once used to treat epilepsy.

Levitin says his experiences in the recording industry now inform his work in the lab. "I was always astonished by how long it takes to make a three-minute song, and how many times you have to record to get it right." He explains that he has to be similarly patient when repeating experiments or redrafting a research paper.

Other scientist performers included neuroscientist and classical guitarist David Sulzer, known on stage as Dave Soldier, and geneticist Pardis Sabeti, of Boston-based band Thousand Days. Among the professional musicians was guitarist Lenny Kaye, who has recorded with punk singer Patti Smith, and Twisted Sister's Dee Snider.

Wainwright held nothing back in his set. He professed he "failed every science course [he] ever took", but supported the charity gig because "scientists have become the new oppressed people, and I'm always there for the oppressed." Roxanne Khamsi is news editor at Nature Medicine in New York.

\title{
Flashes of cosmic brilliance
}

Tim Otto Roth's minimalist art installation reflects the complexity of cosmic radiation, explains Martin Kemp.

Daylight is fading over a grass-covered field. Looking like extra-large bee hives, 252 white cabins on short stilts are regularly distributed across its vast expanse of 44,000 square metres. A larger 'house' occupies the space of the central 4 units. At the centre of each square block of 16 cabins is set a two-tier, highly reflective, cylindrical device.

As darkness envelops the array, shortlived flashes of light erupt unpredictably from the 16 devices. Sometimes one launches alone. At other times they evoke straight lines, or neat clusters. Or just flicker here and there. The flashes are so rapid and apparently random that our eyes search in vain for a repeated pattern. Logic appears to be defied. What are we witnessing? A high-tech scientific experiment? Some sci-fi setting for a war of the worlds? An evocation of a night-time bombing raid?

We are, in fact, watching a minimaliststyle art installation by German artist Tim Otto Roth. His Cosmic Revelation (see http:// tinyurl.com/cosrev) presents a sensory experience of the cosmic radiation detected by the KASCADE project at the Centre for Elementary Particle and Astroparticle Physics at the Karlsruhe Institute of Technology, Germany. Roth's installation is both an artwork and an act of science communication. And it interacts in an unexpected and compelling way with the systematic recording of cosmic phenomena.

The cabins contain detectors for the elementary particles that move at speeds close to that of light and crash incessantly into our atmosphere. These multiple collisions initiate cascades of millions of new particles, and KASCADE measures those that have energies

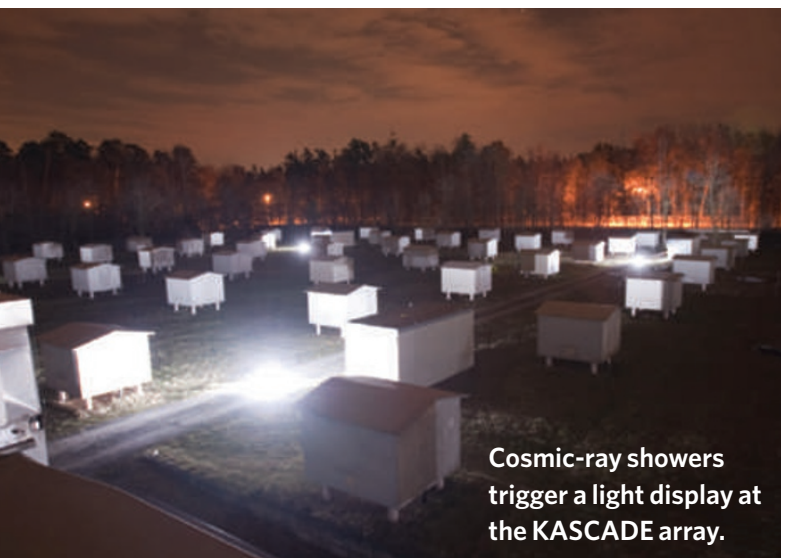

the particles' energies are transmitted back into the space through which they have cascaded.

When Roth categorizes his work as minimalist, he is referring to the reductive mode in art and music that arose in the late 1960s. It relies on the stripping down of a composition into basic units, often regularly repeated and frequently mathematical in form. From the repetition and regularity, paradoxical variousness and complexity emerge when the viewer moves or the lighting changes. Reflective surfaces are particularly favoured.

The generation of complexity from of around $10^{14}-10^{17}$ electronvolts $(\mathrm{eV}$; one $\mathrm{eV}$ is equivalent to the energy acquired by an electron falling through a difference in potential of one volt). Together with other detector units distributed across the research centre, the KASCADEGrande experiment can detect energies as high as $10^{18} \mathrm{eV}$. The results are recorded in a central laboratory by neat grids of LEDs. KASCADE's scientists can view the topographical display of

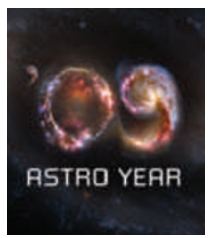
data received by all 252 cabins live on the Internet.

Roth's 16 strobe-light units are in themselves pieces of high-tech sculpture that come into their own as beautiful objects during daylight hours. A 1,500-watt linear flash tube in each unit is protected from the weather by the upper disc. A convex mirror is set into the lower disc so as to project the pulses of light radially from the cylinder's open sides.

These 'cosmic mirrors' carry the visual outputs of the laboratory apparatus back into the field of the outdoor detectors, transforming the energies of the unseen into wavelengths accessible to human sight. Thus transformed, simplicity parallels those physical phenomena that fall under the embrace of chaos theory, and mirrors the reverse processes of measurement, as when the unpredictable complexity of the particle showers are first trapped in the grids of detectors and then registered in the LED arrays in the lab. The chaos of the flashes in Roth's apparatus is placed in a subtle dialogue with both the phenomenon itself and the manner of its recording.

The ancient followers of Pythagoras characterized the harmonics of cosmic energy as the "music of the spheres". The mathematical foundations lay in the proportional system of the musical scale and in the perfections of Euclidean geometry. Here, by contrast, we stand as witnesses to the chaotic drumbeats of cosmic radiation. The new music is that of quantum mechanics and complexity — probabilistic rather than deterministic. A new art is encoding a new science.

Martin Kemp is emeritus professor in history of art at the University of Oxford, Oxford, UK.

See also page 847 and www.nature.com/astro09. 